\title{
Improvement of the CORS method for Cepheids radii determination based on Strömgren photometry
}

\author{
A. Ruoppo ${ }^{1,2}$, V. Ripepi ${ }^{1}$, M. Marconi ${ }^{1}$, and G. Russo ${ }^{2}$ \\ 1 INAF - Osservatorio Astronomico di Capodimonte, via Moiariello 16, 80131 Napoli, Italy \\ e-mail: [ruoppo; marcella]@na.astro.it \\ 2 Dipartimento di scienze fisiche università di Napoli Federico II, Complesso Monte S.Angelo, 80126 Napoli, Italy \\ e-mail: ale@na.infn.it; e-mail: guirusso@unina.it
}

Received 18 December 2003 / Accepted 16 April 2004

\begin{abstract}
In this paper we present a modified version of the CORS method based on a new calibration of the Surface Brightness function in the Strömgren photometric system. The method has been tested by means of synthetic light and radial velocity curves derived from nonlinear pulsation models. Detailed simulations have been performed to take into account the quality of real observed curves as well as possible shifts between photometric and radial velocity data. The method has been then applied to a sample of Galactic Cepheids with Strömgren photometry and radial velocity data to derive the radii and a new PR relation. As a result we find $\log R=(1.19 \pm 0.09)+(0.74 \pm 0.11) \log P(\mathrm{rms}=0.07)$. The comparison between our result and previous estimates in the literature is satisfactory. Better results are expected from the adoption of improved model atmosphere grids.
\end{abstract}

Key words. stars: distances - stars: fundamental parameters - Cepheids

\section{Introduction}

Classical Cepheids are the cornerstone of the extragalactic distance scale. Thanks to their characteristic Period-Luminosity (PL) and Period-Luminosity-Color (PLC) relations they are traditionally used to derive the distances to Local Group galaxies, and (with the advent of space observations) to external galaxies distant up to about $25 \mathrm{Mpc}$ (targets of a Hubble Space Telescope Key Project, see Freedman et al. 1997, 2001). As primary indicators they are used to calibrate a number of secondary distance indicators (see e.g., Freedman et al. 2001) reaching the region of the so called Hubble flow where the Hubble law can be applied and an estimate of the Hubble constant can be derived.

Moreover, the comparison between Cepheid physical parameters (stellar mass, luminosity, chemical composition) based on evolutionary and pulsation models supplies the unique opportunity to pin point the occurrence of deceptive systematic errors (Bono et al. 2001a; Moskalik 2000) on the Cepheid distance scale.

In particular radius determinations are important to constrain both the intrinsic luminosity, through the application of the Stefan-Boltzmann law, provided that an effective temperature calibration is available, and the stellar mass, by adopting a Period-Mass-Radius relation (e.g., Bono et al. 2001).

Send offprint requests to: V. Ripepi, e-mail: ripepi@na.astro.it
Many investigations have been devoted during the last decade to the derivation of accurate Period-Radius (PR) relations for Classical Cepheids both from the empirical (see e.g., Laney \& Stobie 1995; Gieren et al. 1998; Ripepi et al. 1997) and the theoretical (Bono et al. 1998; Marconi et al. 2003) point of view.

Empirical Cepheid radii are generally derived either by means of the Baade Wesselink (BW) method (Moffet \& Barnes 1987; Ripepi et al. 1997; Gieren et al. 1998, just to list a few examples) both in the classical form and in subsequent modified versions, or with interferometric coupled with trigonometric parallaxes techniques (Nordgren et al. 2000; Lane et al. 2002).

The latter method is more direct and less model dependent but up to now it has been applied only to a limited number of stars. On the other hand, the different versions of the BW technique can be applied to relatively large Cepheid samples but require both accurate photometric and radial velocity data.

A powerful modification of the BW technique is the so called CORS method (Caccin et al. 1981), which has the advantage of taking into account the whole light curve rather than selecting phase points at the same color (as in the classical BW implementation), but relies on the adoption of an accurate Surface Brightness (SB) calibration.

Originally Sollazzo et al. (1981) adopted the empirical SB photometric calibration in the Walraven system provided by Pel (1978). More recently Ripepi et al. (1997) modified the method, by adopting the empirical calibration of the reduced surface brightness $F_{V}$ as a function of $(V-R)$ 
provided by Barnes et al. (1976). This modified version of the CORS method was tested, for different colors selections, through the application to synthetic light and radial velocity curves based on nonlinear convective pulsation models (Ripepi et al. 2000).

The recent release of new Cepheid data in the Strömgren photometric system (Arellano-Ferro et al. 1998), and the known sensitivity of intermediate band colors to stellar physical parameters (e.g. gravity and effective temperature) suggested us to investigate the possibility of extending the CORS method to the Strömgren filters.

To this purpose we have derived, in this system, a SB calibration based on model atmosphere tabulations. In this paper we present a modified version of the CORS method based on this new calibration and the application to a sample of Galactic Cepheids.

The organization of the paper is the following: in Sect. 2 we summarize the assumptions and the philosophy of the traditional CORS method; in Sect. 3 we introduce the modified CORS method based on the new SB calibration; in Sect. 4 we test the new method by means of pulsation models; in Sect. 5 we apply the method to a sample of Galactic Cepheids and present the comparison with the literature; in Sect. 6 our final results concerning the PR relation for Galactic Cepheids are shown and the theoretical fit of observed light and radial velocity variations for Cepheid Y Oph is used as an additional check. Some final remarks close the paper.

\section{The original CORS method}

In this section we briefly outline the assumptions and the main features of the CORS method in order to better understand what follows.

The CORS method (Caccin et al. 1981) starts from the definition of the surface brightness:

$S_{V}=V+5 \cdot \log \alpha$

where $\alpha$ is the angular diameter of the star. For a variable star, Eq. (1) is valid for the whole pulsational cycle, so that differentiating it with respect to the phase $(\phi)$, multiplying by a color index $\left(C_{i j}\right)$ and integrating over the whole cycle, one obtains:

$q \int_{0}^{1} \ln \left\{R_{0}-k P \int_{\phi_{0}}^{\phi} v\left(\phi^{\prime}\right) \mathrm{d} \phi^{\prime}\right\} C_{i j}^{\prime} \mathrm{d} \phi-B+\Delta B=0$

where $q=\frac{5}{\ln 10}$

$B=\int_{0}^{1} C_{i j}(\phi) m_{V}^{\prime}(\phi) \mathrm{d} \phi$

$\Delta B=\int_{0}^{1} C_{i j}(\phi) S_{V}^{\prime}(\phi) \mathrm{d} \phi$

where $P$ is the period, $v$ the radial velocity and $k$ is the radial velocity projection factor which relates radial to pulsation velocity $\left(R^{\prime}(\phi)=-k \cdot P \cdot v(\phi)\right)$. The typical value for $k$ is 1.36 (see discussion in Ripepi et al. 1997).
From Eq. (2) we can evaluate the radius $R_{0}$ at an arbitrary phase $\phi_{0}$ (usually taken at the minimum of the radial velocity curve), whereas the mean radius is obtained by integrating twice the radial velocity curve.

The $B$ term can be easily calculated from observed light and color curves. On the contrary, the $\Delta B$ term, which includes the Surface Brightness, is not directly observable. By neglecting it in Eq. (2), we obtain the pure Baade-Wesselink method (see Caccin et al. 1981). However, Sollazzo et al. (1981) and Ripepi et al. (1997, hereinafter RBMR) demonstrated that the inclusion of $\Delta B$ improves the accuracy of radius estimates, provided that $S_{V}$ is evaluated at each pulsation phase.

\section{An improvement of the CORS method based on the Strömgren photometry}

\subsection{Evaluation of the $\Delta B$ term}

As outlined in the previous section, the inclusion of $\Delta B$ improves the accuracy of radius estimates. In this section we present a new good approximation for this term.

As discussed by Onnembo et al. (1985), if the quasi-static approximation $(\mathrm{QSA})^{1}$ is assumed for Cepheid atmospheres, any photometric quantity can be expressed as a function of effective temperature and gravity $\left(T_{\text {eff }}, g_{\text {eff }}\right)$; Then we can write:

$S_{V}=S_{V}\left(T_{\mathrm{eff}}, g_{\mathrm{eff}}\right)$

and

$c_{i j}=c_{i j}\left(T_{\text {eff }}, g_{\text {eff }}\right)$

$c_{k l}=c_{k l}\left(T_{\mathrm{eff}}, g_{\mathrm{eff}}\right)$

where $S_{V}$ is the surface brightness in the visual band, and $c_{i j}=$ $\left(m_{i}-m_{j}\right), c_{k l}=\left(m_{k}-m_{l}\right)$ are two arbitrary colors.

If the last two equations are invertible, i.e the Jacobian $J\left(T_{\text {eff }}, g_{\text {eff }} \mid C_{i j}, C_{k l}\right) \neq 0$, then we can invert Eqs. (6), obtaining:

$T_{\text {eff }}=T_{\text {eff }}\left(c_{i j}, c_{k l}\right)$

$g_{\mathrm{eff}}=g_{\mathrm{eff}}\left(c_{i j}, c_{k l}\right)$

and hence

$S_{V}=S_{V}\left(c_{i j}, c_{k l}\right)$.

We point out that Eqs. (6) do not admit a general solution over the whole parameter space, given that the same colors can be obtained for different pairs of $T_{\text {eff }}$ and $g_{\text {eff }}$. This notwithstanding it is possible to find a local solution, that is a solution valid only in the parameter space defined by Cepheids.

A potentially advantageous choice for the colors $c_{i j}, c_{k l}$ could be represented by the Strömgren reddening free

\footnotetext{
${ }^{1}$ We recall that a sufficient condition for the validity of the QSA is that the atmosphere of the pulsating star can be described, at any time, by a classical hydrostatic, plane parallel model in radiative/convective equilibrium and local-thermodynamic-equilibrium (LTE), identified by the effective temperature $T_{\text {eff }}$ and by the effective gravity $g_{\text {eff }}=$ $\frac{G M}{R^{2}}+\frac{\mathrm{d}^{2} R}{\mathrm{~d} t^{2}}$.
} 


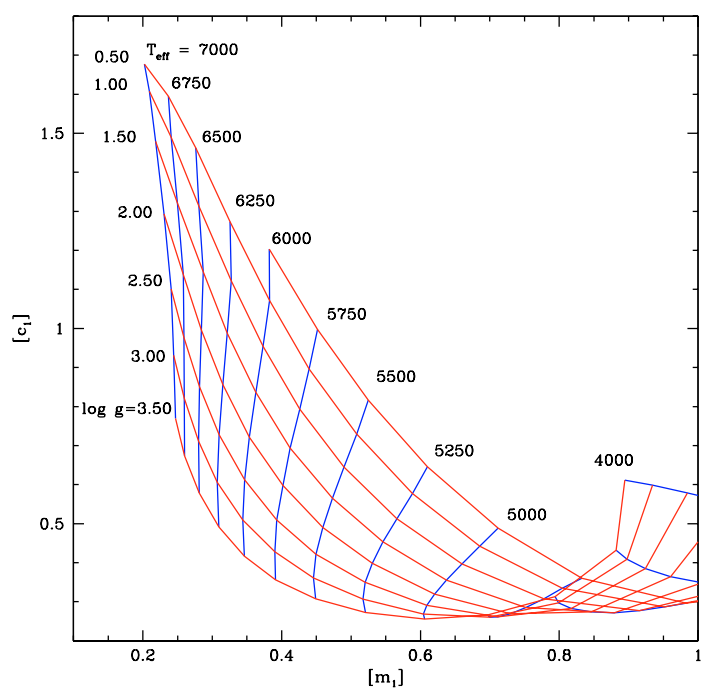

Fig. 1. A grid of lines at constant $T_{\text {eff }}$ and constant $\log g$ in the theoretical $\left[m_{1}\right],\left[c_{1}\right]$ plane.

indexes $\left[m_{1}\right]$ and $\left[c_{1}\right]$ defined as follows (Crawford \& Mandwewala 1976):

$\left[m_{1}\right]=m_{1}+0.33(b-y)$

$\left[c_{1}\right]=c_{1}-0.16(b-y)$

where the coefficients 0.33 and -0.16 are suitable for F supergiant stars (Gray 1991).

In this context, an interesting possibility to derive Eqs. (7), consists in using grids of theoretical colors, calculated by means of model atmospheres, to obtain $T_{\text {eff }}$ and $g_{\text {eff }}$ as a function of $\left[m_{1}\right]$ and $\left[c_{1}\right]$.

To verify this possibility, we have adopted the grids of theoretical colors by Castelli et al. (1997a,b, hereinafter C97a,b). The theoretical grid (constant $T_{\text {eff }}$ and $\log g$ ) in the $\left[m_{1}\right],\left[c_{1}\right]$ plane is shown in Fig. 1. This figure suggests that for $0.50 \leq$ $\log g \leq 3.50$ and $5000 \mathrm{~K} \leq T_{\text {eff }} \leq 7000 \mathrm{~K}$ there is a one-to-one correspondence between a point in the $\left[m_{1}\right],\left[c_{1}\right]$ plane and the corresponding $T_{\text {eff }}$ and $\log g$ values. Thus, in principle, in this color range it is possible to invert Eqs. (6) and, in turn, to derive an expression for Eq. (8).

Before proceeding, it is important to verify if the location of theoretical grids in the $\left[m_{1}\right],\left[c_{1}\right]$ plane is consistent with the one occupied by real Cepheid data. To this aim, we have overplotted in Fig. 1 the color-color $\left[m_{1}\right],\left[c_{1}\right]$ loop for all the stars in our sample (see Sect. 5.1). Figure 2 shows the resulting comparison for three stars (FF Aql, FN Aql, U Aql) of our sample, characterized by short and intermediate periods. The figure shows that the color-color loops for the selected stars are completely included in the theoretical grids. The same test has been performed for the three stars W Sgr, WZ Sgr, SV Vul, which have longer periods. As showed by Fig. 3, in this case the color-color loops for the selected Cepheids lies outside (at low gravity and low effective temperature) of the region covered by theoretical grids. This unexpected result has to be taken into account when applying our method to such long period stars. An explanation for this model limitation is beyond the scopes of

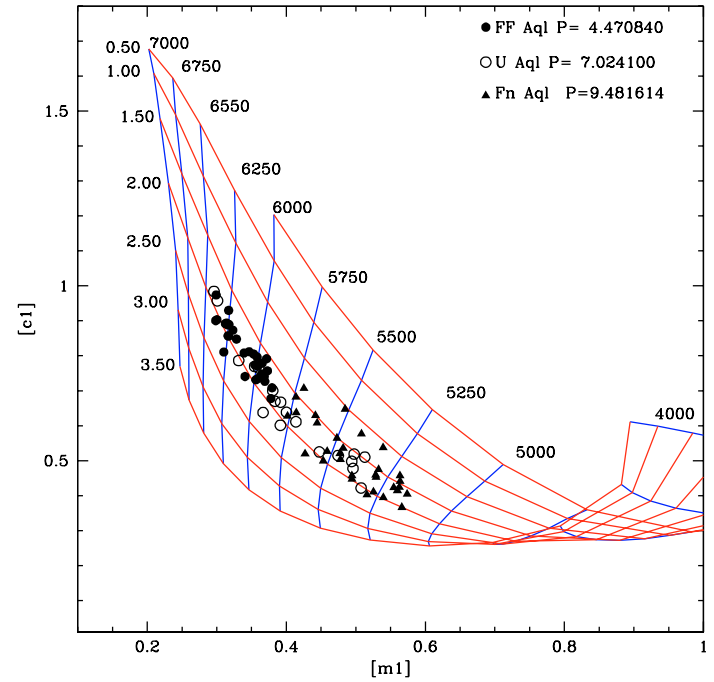

Fig. 2. Theoretical grids in the $\left[m_{1}\right],\left[c_{1}\right]$ plane, compared with the empirical loops of three stars with period shorter than $10 \mathrm{~d}$.

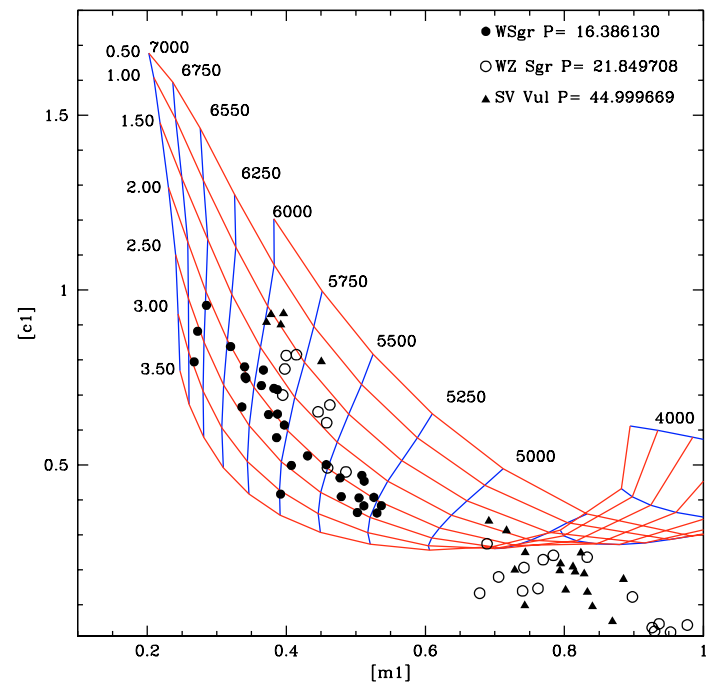

Fig. 3. The same as in Fig. 2 but for three Cepheids with period longer than $15 \mathrm{~d}$.

present paper but it is an important issue worth to be addressed in a future work.

\subsubsection{Derivation of $S_{V}$}

As shown in the previous section, the first step in the construction of a new, based on Strömgren photometry, version of the CORS method, is the formulation of $S_{V}$ in the form of Eq. (8). To this aim, we have first to find relations in the form of Eqs. (7) starting from theoretical grids. This has been achieved by means of a 4th degree polynomial fit to the theoretical grid (least square fit). The results of the polynomial approximations are reported in Appendix A, and showed in Figs. 4 and 5 for $\log T_{\text {eff }}$ and $\log g_{\text {eff }}$ respectively. The rms of the fits are: $0.0018 \mathrm{dex}$ for $\log T_{\text {eff }}$ and 0.1 for $\log g_{\text {eff }}$.

On the basis of the calculated relations we are now in the position to estimate the surface brightness from the expression:

$S_{V}=$ const. $-10 \log T_{\text {eff }}-B C\left(T_{\text {eff }}, g_{\text {eff }}\right)$ 


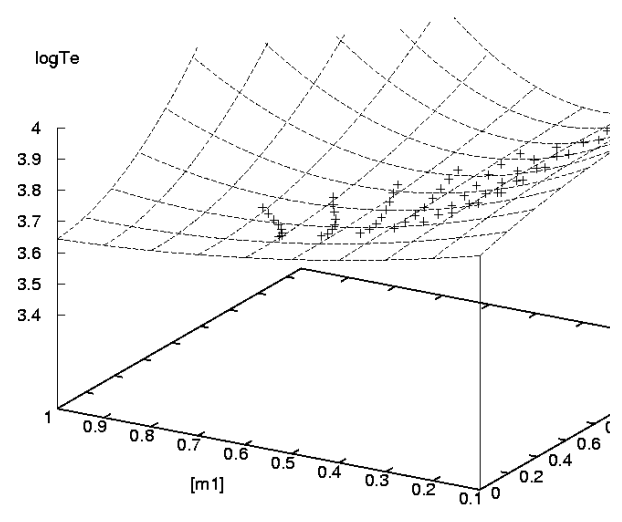

Fig. 4. $\log T_{\text {eff }}$ as a function of $\left[m_{1}\right],\left[c_{1}\right]$ obtained by a 4 th degree polynomial fit of theoretical data (crosses) by C97a,b.

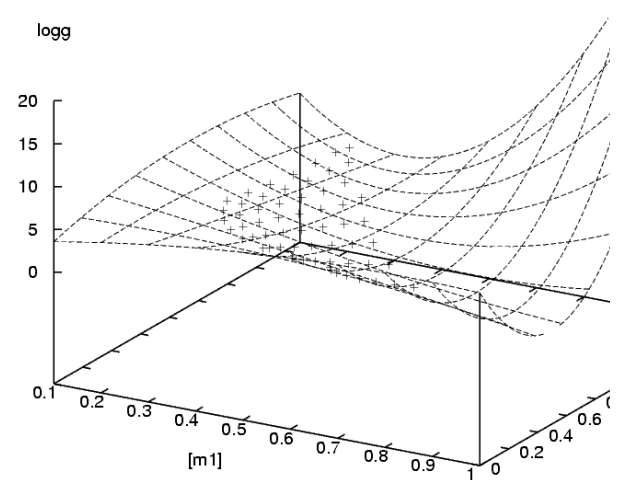

Fig. 5. The same as in Fig. 4 but for $\log g$.

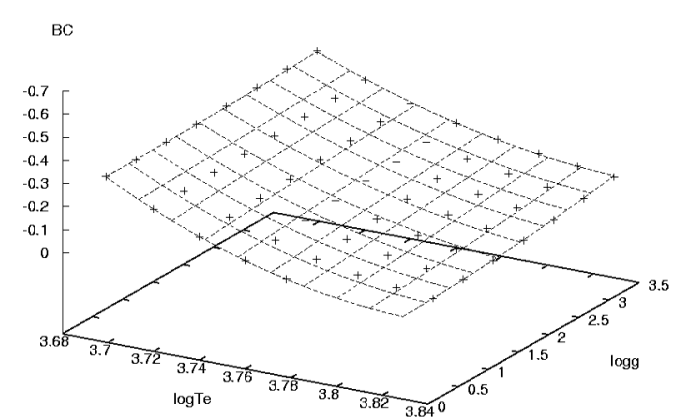

Fig. 6. $B C$ as a function of $\log T_{\mathrm{e}}, \log g$ obtained by a 4 th degree polynomial fit of theoretical data (crosses) by C97a,b.

where $B C$ is the Bolometric Correction, derived as a function of effective temperature and gravity $\left(B C=B C\left(T_{\text {eff }}, g_{\text {eff }}\right)\right)$. This function can be easily obtained using again a 4 th degree polynomial fit to the theoretical grids. The resulting equation is also reported in Appendix A and shown in Fig. 6. Note that the rms is only 0.003 mag.

The procedure outlined above allowed us to achieve our goal, i.e. to derive an analytic, although approximated, expression of $S_{V}=S_{V}\left(\left[m_{1}\right],\left[c_{1}\right]\right)$, and, in turn, to calculate the $\Delta B$ term, which allows us to apply the CORS method, in its more general formulation, for data in the Strömgren photometric system.

\section{Test of the revised CORS method}

To verify the accuracy of the new approximation, we have applied the CORS method (with the new $S_{V}$ calibration) to synthetic light, color and radial velocity curves predicted by Cepheid full amplitude, nonlinear, convective models. The advantages of testing the method by means of pulsation model curves, rather than empirical data, are the following:

1. for pulsation models, bolometric light curves are transformed into the observational bands using the same model atmosphere tabulations (C97a,b) we have used to derive the surface brightness calibration;

2. pulsation models provide a set of equally well sampled curves covering wide period and effective temperature ranges;

3. for pulsation models the radius and intrinsic luminosity information are available.

In the following we describe in detail how the models have been used to test the method.

\subsection{The synthetic curves}

To test both the accuracy and the consistency of the new CORS method we have adopted the pulsation observables predicted by hydrodynamical models of classical Cepheids. A detailed discussion on the physical assumptions adopted to calculate these models can be found in Bono et al. (1999, BMS99; 2002). Among the different sequences of nonlinear models computed by BMS99 we have selected canonical models ${ }^{2}$ at solar chemical composition $(Y=0.28, Z=0.02)$ and stellar masses ranging from 5 to $9 M_{\odot}$. At fixed stellar mass, we generally chose three models which are located in the middle of the instability strip as well as close to the blue and the red edge. The period of selected models roughly ranges from 3.5 to 62 days. The input parameters $\left(M, T_{\text {eff }}, L\right)$, the computed radius and the pulsational period are summarized in Table 1.

Theoretical observables have been transformed into the observational plane by adopting the bolometric corrections $(B C)$ and the color-temperature relations by $\mathrm{C} 97 \mathrm{a}, \mathrm{b}$. We assumed $M_{\mathrm{Bol}}(\odot)=4.62 \mathrm{mag}$ and adopted atmosphere models computed by neglecting the core overshooting and for a fixed value of the microturbolence velocity $\xi=2 \mathrm{~km} \mathrm{~s}^{-1}$.

For each pulsation model we have derived the $V$ light curve, three color curves, namely in $(b-y),\left[m_{1}\right]$ and $\left[c_{1}\right]$ and the radial velocity curve. Figure 7 shows these curves for models with $M=9 M_{\odot}, T_{\text {eff }}=4500 \mathrm{~K}$, and $M=7 M_{\odot}, T_{\text {eff }}=5300 \mathrm{~K}$.

\subsection{Application to theoretical models}

As a first test, we have applied the method to "perfect" light, color and radial velocity curves, i.e. the curves directly obtained from the models which, of course, do not show random

\footnotetext{
${ }^{2}$ Canonical models are the ones constructed by adopting a massluminosity relation based on evolutionary computations which neglect convective core overshooting during hydrogen burning phases (Castellani et al. 1992).
} 
Table 1. Physical properties of the selected Cepheid models.

\begin{tabular}{rrccrr}
\hline \hline Model & $\begin{array}{r}\text { Mass } \\
M_{\odot}\end{array}$ & $\begin{array}{c}\text { Luminosity } \\
\log L / L_{\odot}\end{array}$ & $\begin{array}{c}T_{\text {eff }} \\
\mathrm{K}\end{array}$ & $\begin{array}{r}\text { Radius } \\
R_{\odot}\end{array}$ & $\begin{array}{r}\text { Period } \\
\text { Days }\end{array}$ \\
\hline Mod.1 & 5 & 3.07 & 5800 & 34.3 & 3.5091 \\
Mod.2 & 5 & 3.07 & 5600 & 36.7 & 3.9412 \\
Mod.3 & 6.25 & 3.42 & 5400 & 58.6 & 7.5842 \\
Mod.4 & 6.25 & 3.42 & 5100 & 65.2 & 8.7060 \\
Mod5 & 7 & 3.65 & 5300 & 81.0 & 12.0904 \\
Mod.6 & 7 & 3.65 & 5000 & 91.3 & 14.7582 \\
Mod7 & 7 & 3.65 & 4800 & 97.3 & 16.8322 \\
Mod.8 & 9 & 4.0 & 4900 & 185.7 & 46.5687 \\
Mod.9 & 9 & 4.0 & 4700 & 204.1 & 53.9378 \\
Mod.10 & 9 & 4.0 & 4500 & 220.6 & 62.3650 \\
\hline
\end{tabular}
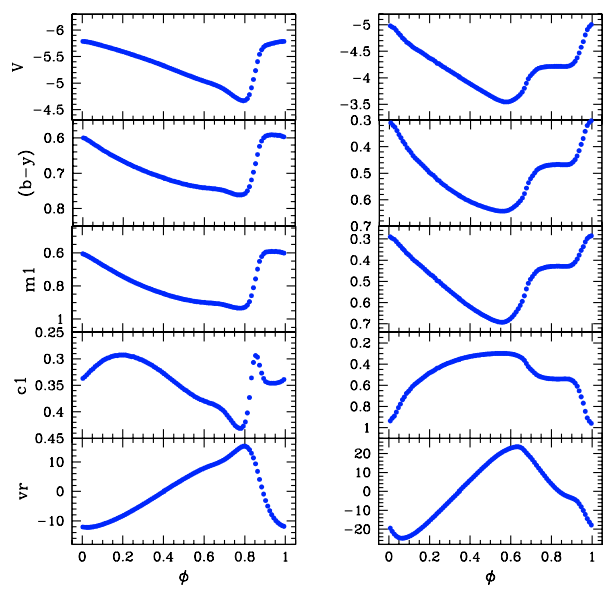

Fig. 7. Variations along a full pulsation cycle of magnitude, color and radial velocity for models with $M=9 M_{\odot}$ and $T_{\text {eff }}=4500 \mathrm{~K}$ (left) and $M=7 M_{\odot}$ and $T_{\text {eff }}=5300 \mathrm{~K}($ right $)$.

errors. This test allows us to verify whether or not our calibration of the surface brightness is intrinsically correct. If this is the case we do not expect a large discrepancy between the calculated radii and the "true" (theoretical) ones.

To perform this comparison we have evaluated the radius of each model in the two different approximations:

- without the $\Delta B$ term (Baade-Wesselink approximation);

- with the $\Delta B$ estimated as described in Sect. 3.

The results of this test are summarized in the first four columns of Table 2. We note that, the application to models with long period is justified by the fact that the bolometric light curves of pulsation models have been transformed into the observational bands by using the same model atmospheres by C97a,b. Therefore, at variance with the observed long period Cepheids, the model color-color loops are always consistent with C97a,b's grids.

The same result is also shown in Fig. 8, which suggests that our calibration of the surface brightness is intrinsically correct with the discrepancy between "computed" and "theoretical" Cepheid radii being around $1 \%$. We also note that the
Table 2. Radii estimated with the two different approximations, i.e with and without the $\Delta B$ term (respectively Cols. 2, 3), using model predictions (Col. 4), and from modified synthetic curves in the case of good (Col. 5) and fair (Col. 6) data respectively.

\begin{tabular}{cccccc}
\hline \hline Models & $R_{\text {without } \triangle B}$ & $R_{\Delta B}$ & $R_{\text {teo }}$ & $R_{\triangle B}^{1}$ & $R_{\Delta B}^{2}$ \\
& $\frac{R}{R_{\odot}}$ & $\frac{R}{R_{\odot}}$ & $\frac{R}{R_{\odot}}$ & $\frac{R}{R_{\odot}}$ & $\frac{R}{R_{\odot}}$ \\
\hline Mod. 1 & 34.7 & 34.8 & 34.3 & 37.5 & 36.3 \\
Mod. 2 & 36.7 & 36.6 & 36.7 & 36.7 & 38.6 \\
Mod. 3 & 59.1 & 59.0 & 58.6 & 59.5 & 60.9 \\
Mod. 4 & 64.6 & 65.7 & 65.2 & 63.1 & 73.1 \\
Mod. 5 & 82.1 & 82.2 & 81.0 & 81.3 & 78.0 \\
Mod. 6 & 92.4 & 92.3 & 91.3 & 90.6 & 94.0 \\
Mod. 7 & 98.6 & 98.5 & 97.3 & 99.4 & 99.6 \\
Mod. 8 & 190.3 & 188.8 & 185.7 & 185.8 & 153.4 \\
Mod. 9 & 206.9 & 206.5 & 204.1 & 202.0 & 203.1 \\
Mod. 10 & 223.1 & 223.4 & 220.6 & 187.7 & 177.0 \\
\hline
\end{tabular}

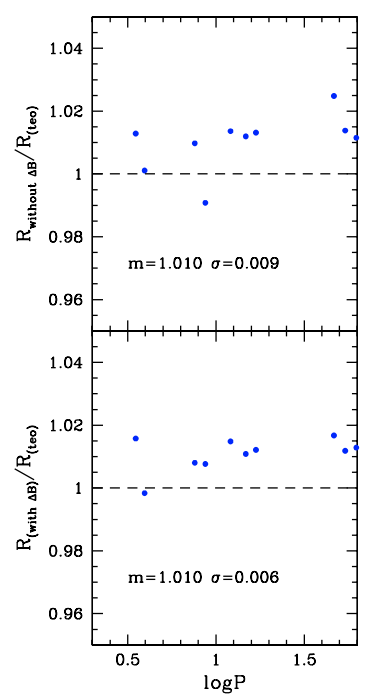

Fig. 8. Ratio between "computed" and "theoretical" radii as a function of the logarithmic period. The bottom panel displays the radius evaluation based on the revised CORS method, while the top one the radius evaluations based on the pure BW method.

inclusion of the $\Delta B$ term only slightly improves the agreement with predicted radii, producing a small reduction of the scatter around the mean (see labelled values in Fig. 8).

\subsection{Stability tests}

Once verified that the new calibration of the surface brightness is intrinsically correct, we are in the position to estimate the statistical error in the radius determination due to uncertainties in the measurements, i.e. to test the sensitivity of our technique to the accuracy of observed light, color and radial velocity curves. To this aim, we have transformed the synthetic curves for models in Table 1 by adopting the following steps:

1. we have reduced the number of phase points to the typical value for observations, i.e. 30-35; 


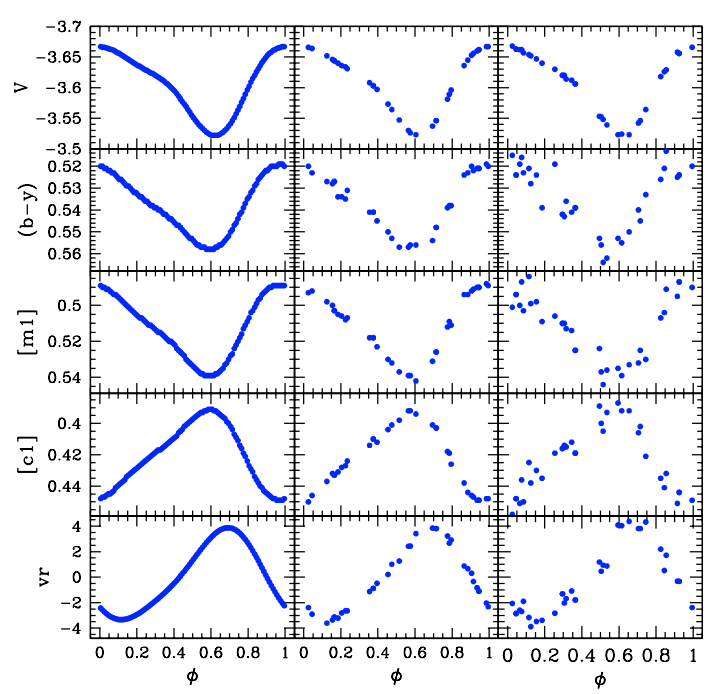

Fig. 9. Light, color and radial velocity curves for the model (Mod. 4) with $M=6.25 M_{\odot}$ and $T_{\text {eff }}=5100 \mathrm{~K}$. The left panels show the pure model curves; the middle and right ones the modified curves in the case of good and fair data respectively.

2. we have extracted phases randomly;

3. we have added Gaussian errors, considering the cases:

a) good data $\left(\sigma_{V}=0.02 \mathrm{mag}, \sigma_{\left[m_{1}\right]}=0.04 \mathrm{mag}, \sigma_{\left[c_{1}\right]}=\right.$ $0.04 \mathrm{mag}, \sigma_{R V}=0.25 \mathrm{~km}^{-1}$, number of phase points $\left.=35\right)$;

b) fair data $\left(\sigma_{V}=0.04 \mathrm{mag}, \sigma_{\left[m_{1}\right]}=0.08 \mathrm{mag}, \sigma_{\left[c_{1}\right]}=\right.$ $0.08 \mathrm{mag}, \sigma_{R V}=0.5 \mathrm{~km}^{-1}$, number of points $=30$ ).

As an example, Fig. 9 shows the synthetic light, color and radial velocity curves obtained for model Mod. 4 (see Table 1). Left panels show the pure model curves, whereas middle and right panels show the modified curves in the two cases a) and b) respectively.

We then applied the new CORS method to the whole set of modified synthetic curves. The resulting radii are reported in the last two columns of Table 2, whereas in Fig. 10 we plot the ratio between "computed" and "theoretical" radii as a function of the logarithmic period. In particular, top and bottom panels show the CORS solutions without and with $\Delta B$ respectively for the cases a) (left) and b) (right). Figure 10 seems to show that including the $\Delta B$ term does not improve much the results, on the contrary, the scatter in case a) worsens. However, this disagreeable occurrence is only apparent. In fact, by excluding "Mod. 8" and "Mod. 10" that show a very peculiar morphology (a sharp bump) of the $\left[c_{1}\right]$ color curve (see figure Fig. 7 for "Mod. 10"), which makes difficult the fit and the $\Delta B$ calculation, the average uncertainties on the radius estimation fall to $3.5 \%$ and $5 \%$ in cases a) and b) respectively (including the $\Delta B$ term).

These numbers represent a useful lower limit for the error associated with radius determination obtained by the CORS version developed in this paper.

Another possible source of uncertainty in the radius determination is the misalignment between light or color curves, and the radial velocity one. In fact, the photometric and radial velocity data are rarely collected simultaneously. This occurrence could introduce a shift in phase $\Delta \phi$ between the two different
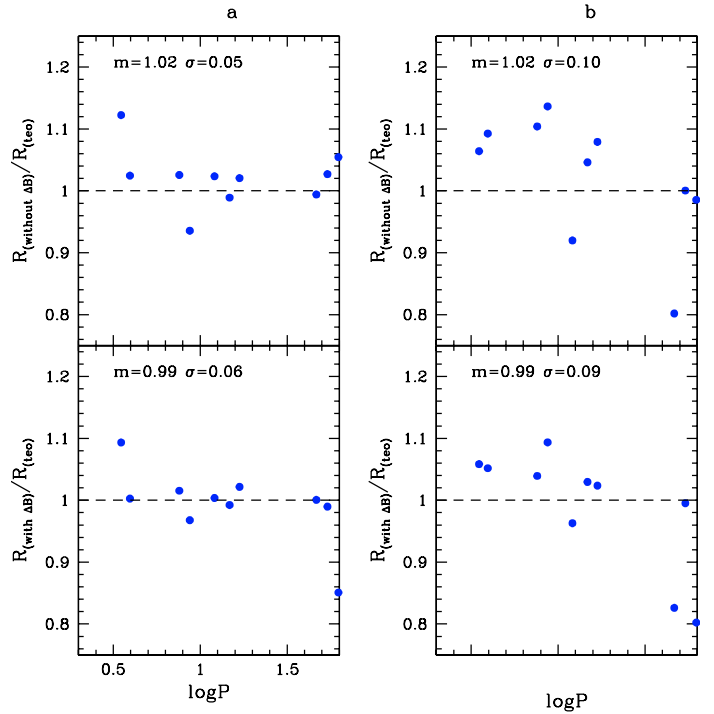

Fig. 10. Ratio between "computed" and "theoretical" radii as a function of the logarithmic period. In the left panels a) the radius is computed using modified curves that simulate good quality, adopting either the revised CORS method (bottom) or the pure BW one (top). In the right panels b) similar plots are shown for radii computed from modified curves that simulate fair quality data.

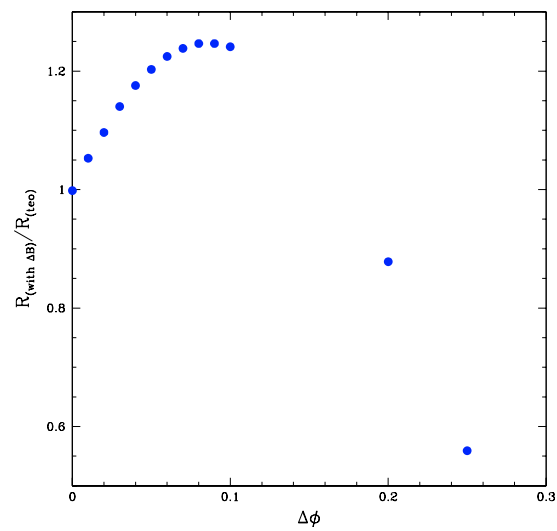

Fig. 11. Ratio between the "computed" and "theoretical" radii as a function of the shift $\Delta \phi$ for the model (mod. 2) with $M=5 M_{\odot}, T_{\text {eff }}=$ $5600 \mathrm{~K}$.

data set. To verify the importance of this shift on the radius determination we have introduced an artificial phase shift in the synthetic radial velocity curves with respect to the photometric ones.

The result of such a test is shown in Fig. 11, where the ratio between "computed" and "theoretical" radii is plotted as a function of the phase shift $\Delta \phi$ for the model Mod. 2. The "computed" radius is larger than the "theoretical" one for $0<\Delta \phi \leq 0.1$, whereas it becomes smaller beyond 0.1 with the effect increasing with the phase shift. Eventually, our program does not converge for $\Delta \phi \geq 0.3$. However, such large phase shifts are never reached when we deal with actual data. In fact, when the temporal distance between photometric and radial velocity data is rather long $(\sim 1000$ cycles $)$, for a typical Cepheid with period $P \sim 10 \pm 5 \times 10^{-5} \mathrm{~d}$, the resulting phase shift is $\Delta \phi \sim 0.05$. As shown in Fig. 11, this value of $\Delta \phi$ is 
sufficient to generate a systematic error on the derived radius of about $17 \%$. This occurrence confirms quantitatively the need to use photometric and radial velocity data as close as possible in time, or otherwise to correct this shift.

\section{Application of the method}

Having tested the capabilities of the new version of the CORS method (see previous section), we are ready to apply it to actual data. In the following we discuss the application to a sample of 52 Galactic Cepheids.

\subsection{The sample}

We searched the literature for an homogeneous sample of Galactic Cepheids with photometric data in the Strömgren system and we selected the 31 pulsators analysed by Arellano Ferro et al. (1998) (AFGR hereafter) supplemented by data for other 21 objects from the papers by Feltz \& McNamara (1980) (FM hereafter) and Eggen $(1985,1996)$ (Eg hereafter).

Concerning radial velocity data, several large and homogeneous datasets are available in the literature. In particular, we have used the catalogues from: Evans (1980) (E hereafter), Gieren (1981) (G hereafter), Barnes et al. (1987, 1988), (BMS hereafter), Coulson et al. (1985) (CCG hereafter), Wilson et al. (1989) (WCB hereafter), Metzger et al. (1993) (MCMS hereafter), Bersier et al. (1994) (BBMD hereafter), Gorynya et al. (1998) (GSSRGA hereafter), Kiss (1998) (K hereafter) and Imbert (1999) (I hereafter).

In general we considered only Cepheids with good photometric and radial velocity curves, i.e. more than 15 phase points and reasonable precision. When measurements by different authors are available for the same stars we have chosen data which have: 1) the largest phase points number and the best precision; 2) the lowest temporal separation with respect to the photometric data. When we have distant data sets, that is differences between radial velocity and photometric curves larger than $\sim 1000$ cycles ( 6 stars, see Table 3 ), we correct the misalignment between this two curves by using different epochs.

In few cases we have merged data from different authors, in order to obtain more sampled radial velocity curves (see Table 3). Our final selected dataset is summarized in Table 3.

\subsection{Peculiar stars}

Some Cepheids in our sample need to be discussed individually:

- for the stars TT Aql, X Cyg, T Mon, RU Sct, Y Sct, WZ Sgr and SV Vul, we can calculate the radius only in the classical Baade-Wesselink approximation (without the $\Delta B$ term), because for these stars the loop in the $\left[m_{1}\right],\left[c_{1}\right]$ plane does not lie completely within the theoretical grids by C97a,b (see paragraph 3.1);

- we have excluded from our sample the Cepheids GI Car, SU Cas, Y Lac, SZ Tau, X Vul for the poor data quality;
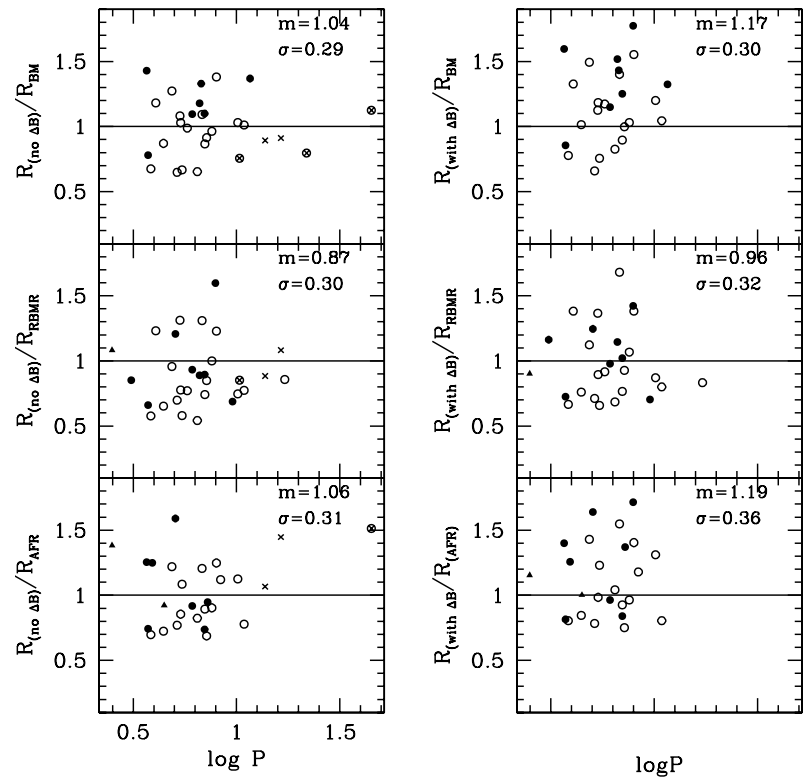

Fig. 12. Comparison of radii obtained in this work $\left(R_{\text {with } \triangle \mathrm{B}}, R_{\mathrm{no}} \Delta \mathrm{B}\right)$ with those derived by other authors (Arellano Ferro \& Rosenzweing (2000) $R_{\mathrm{AFR}}$; Ripepi et al. (1997) $R_{\mathrm{RBMR}}$; Moffett \& Barnes (1987) $R_{\mathrm{BM}}$ ).

- stars DT Cyg, FF Aql, BG Cru and V440 Per are first overtone pulsators (Antonello et al. 1990);

- stars TU Cas, V367 Sct and BQ Ser are double mode Cepheids (Pardo \& Poretti 1997, and references therein);

- stars U Aql, FF Aql, V 496 Aql, $\eta$ Aql, RX Aur, SU Cas, $\delta$ Cep, GI Car, SU Cyg, VZ Cyg, $\zeta$ Gem, X Lac, Y Lac, Z Lac, BG Lac, T Mon, Y Oph, BF Oph, AW Per, Y Sct, S Sge, W Sgr, Y Sgr, WZ Sgr, AP Sgr, V 350 Sgr, SZ Tau, T Vul, U Vul and SV Vul are members of binary systems. Note that this list is the result of a detailed analysis of Szabados's selection (Szabados 2003, and references therein).

\subsection{Comparison with the literature}

In Table 4 we report the radius obtained for all the Cepheids in our sample and, for comparison purposes, we report the literature results renormalized to our projection factor $k$. In particular, from left to right we report for each Cepheid: the name; the period; the CORS radii obtained without and with the $\Delta B$ term $\left(R_{\text {without } \triangle B}\right.$ and $\left.R_{\Delta B}\right)$; the radii obtained by Arellano Ferro \& Rosenzweing (2000) $R_{\mathrm{AFR}}$, Laney \& Stobie (1995) $R_{\mathrm{LS}}$ or Gieren et al. (1998) $R_{\mathrm{GFG}}$, Ripepi et al. (1997) $R_{\mathrm{RBMR}}$. and Moffett \& Barnes (1987) $R_{\mathrm{BM}}$.

In Fig. 12 we have compared our results with those of authors reported in Table 4. As a result we find that our radii are on average larger than the radii obtained by Arellano Ferro \& Rosenzweing (2000) and Moffett \& Barnes (1987), while they are slightly smaller than Ripepi et al. (1997) ones (see labels in Fig. 12). The scatter in this comparisons is rather large $(\sim 30 \%)$; this occurrence could be due to: 1$)$ the inclusion of binary stars in the comparison (different methods use different colours and then binarity could affect differentially the various 
Table 3. The selected Cepheid sample.

\begin{tabular}{|c|c|c|c|c|c|c|c|c|c|}
\hline Cepheid & $\begin{array}{c}\text { Period } \\
\text { days }\end{array}$ & $\begin{array}{c}\text { Photometry } \\
\text { source }\end{array}$ & $\begin{array}{c}\text { Rad. Vel. } \\
\text { source }\end{array}$ & $\begin{array}{c}\Delta(P h-R V) \\
\text { cycles }\end{array}$ & Cepheid & $\begin{array}{c}\text { Period } \\
\text { days }\end{array}$ & $\begin{array}{c}\text { Photometry } \\
\text { source }\end{array}$ & $\begin{array}{c}\text { Rad. Vel. } \\
\text { source }\end{array}$ & $\begin{array}{c}\Delta(P h-R V) \\
\text { cycles }\end{array}$ \\
\hline FF Aql & 4.470840 & AFGR & BMS & 1142 & YOph & 17.126780 & AFGR & GSSRGA & 46 \\
\hline FM Aql & 6.114334 & AFGR & GSSRGA & 89 & AW Per & 6.463589 & FM & GSSRGA & 422 \\
\hline FN Aql & 9.481614 & AFGR & GSSRGA & 56 & V440 Per & 7.572712 & $\mathrm{Eg}$ & GSSRGA & 131 \\
\hline$\eta \mathrm{Aql}$ & 7.176779 & FM & BMS & 413 & CM Sct & 3.916977 & AFGR & M CMS, B & 463 \\
\hline TT Aql & 13.75551 & AFGR & GSSRGA & 81 & EV Sct & 3.090998 & AFGR & BBMD, MCMS & 586 \\
\hline U Aql & 7.024100 & AFGR & BMS & 674 & RU Sct & 19.70062 & AFGR & MCMS & 92 \\
\hline V496 Aql & 6.807164 & AFGR & G & 643 & SS Sct & 3.671280 & AFGR & BMS, G & 1194 \\
\hline V600 Aql & 7.238748 & AFGR & GSSRGA & 156 & V367 Sct & 6.29307 & AFGR & MCMS & 317 \\
\hline RT Aur & 3.728561 & FM & GSSRGA & 216 & Y Sct & 10.341650 & AFGR & BMS & 421 \\
\hline RX Aur & 11.623537 & FM & I & 271 & BQ Ser & 4.316700 & AFGR & GSSRGA & 926 \\
\hline GI Car & 4.431035 & $\mathrm{Eg}$ & G & - & S Sge & 8.382044 & FM & GSSRGA & 209 \\
\hline SU Cas & 1.949322 & FM & GSSRGA & - & AP Sgr & 5.0574269 & AFGR & BMS, G & 864 \\
\hline TU Cas & 2.139298 & FM & GSSRGA & - & BB Sgr & 6.637115 & AFGR & GSSRGA & 166 \\
\hline$\delta$ Cep & 5.366316 & FM & BBMD & 393 & U Sgr & 6.745363 & AFGR & GSSRGA & 81 \\
\hline DT Cyg & 2.499086 & AFGR & GSSRGA & 2203 & V350 Sgr & 5.154557 & AFGR & GSSRGA & 157 \\
\hline SU Cyg & 3.845733 & AFGR & BMS & 1432 & X Sgr & 7.012630 & AFGR & WCB & 759 \\
\hline X Cyg & 16.386130 & AFGR & BBMD & 244 & Y Sgr & 5.773400 & AFGR & BMS, WCB & 885 \\
\hline VZ Cyg & 4.864504 & FM & BBMD & 50 & YZ Sgr & 9.553606 & AFGR & BMS & 419 \\
\hline BG Cru & 3.342503 & $\mathrm{Eg}$ & $\mathrm{E}$ & 943 & W Sgr & 7.595080 & AFGR & BBMD & 542 \\
\hline W Gem & 7.913960 & FM & I & 257 & WZ Sgr & 21.849708 & AFGR & GSSRGA & 50 \\
\hline$\zeta \mathrm{Gem}$ & 10.150780 & FM & BBMD & 179 & SZ Tau & 3.148727 & FM & GSSRGA & - \\
\hline BG Lac & 5.331938 & FM & I & 114 & SV Vul & 44.999660 & AFGR & I & 103 \\
\hline Y Lac & 4.323776 & FM & I & 46 & $\mathrm{~T}$ Vul & 4.435532 & FM & BBMD & 530 \\
\hline Z Lac & 10.88554 & FM & BMS,GSSRGA & 273 & U Vul & 7.990736 & AFGR & GSSRGA & 142 \\
\hline X Lac & 5.444990 & FM & BBMD & 1.9 & X Vul & 6.319562 & FM & GSSRGA & - \\
\hline T Mon & 27.024649 & FM & GSSRGA & - & & & & & \\
\hline BF Oph & 4.067695 & AFGR & BMS, G & 1077 & & & & & \\
\hline
\end{tabular}

Photometry sources: Arellano Ferro et al. (AFGR, 1998); Feltz \& McNamara (FM, 1980) and Eggen (Eg, 1985, 1996).

Radial velocity sources: Evans (E, 1980); Gieren (G, 1981); Barnes et al. (BMS, 1987, 1988); Coulson et al. (CCG, 1985); Wilson et al. (WCB, 1989); Metzger et al. (MCMS, 1993); Bersier et al. (BBMD, 1994); Gorynya et al. (GSSRGA, 1998); Kiss (K, 1998) and Imbert (I, 1999). $\Delta(P h-R V)$ : number of pulsational cycles between photometric and radial velocity curves.

determinations); 2) the use of optical colors. Verifying hypothesis 1) by comparing only the non-binary stars does not make sense because of the small number of "bona fide" single stars in our sample.

Concerning point 2), as well known in the literature (Laney \& Stobie 1995; Gieren et al. 1997), radii obtained from NIR data are more precise than the ones obtained by using optical colors. Unfortunately, most works with radii determinations from NIR data deal with southern Cepheids and we have very few stars in common (see Col. 7 of Table 4 for the comparison).

Recently it has become possible to derive accurate stellar radii from the angular diameters measured with interferometric techniques (see Nordgren et al. 2000 and Lane et al. 2002) combined with Hypparcos parallaxes. The comparison with these measurements represents a useful test for our results. In Table 5 we compare with our results the radii obtained by Nordgren et al. (2000) and Lane et al. (2002), with the quoted interferometric techniques, for the stars in common with our sample, namely $\eta \mathrm{Aql}, \delta$ Cep and $\zeta$ Gem. In particular, from left to right, we report: the name of the star; the radius $\left(R_{I}\right)$ obtained by means of interferometric techniques ( $\delta$ Cep from Nordgren et al. 2000 and $\eta$ Aql and $\zeta$ Gem from Lane et al. 2002); our radius in the two different approximations, without $\left(R_{\text {without } \triangle \mathrm{B}}\right)$ and with $\left(R_{\mathrm{with}} \Delta B\right)$ the $\Delta B$ term respectively. The interferometric radii, reported in Table 5, are corrected for the different $k$ projection factors. So we obtain the radii $61.8 \times 1.36 / 1.43=58.8 R_{\odot}, 45 \times 1.36 / 1.31=47 R_{\odot}$ and $66.7 \times 1.36 / 1.43=63.4 R_{\odot}$ for $\eta \mathrm{Aql}, \delta$ Cep and $\zeta \mathrm{Gem}$, respectively. We notice that the agreement between our results and the interferometric ones is very good for $\eta$ Aql and $\delta$ Cep, whereas some discrepancy is found for $\zeta$ Gem. However, by assuming a typical error of $\sim 10 \%$ (see discussion in Sect. 7 ) 
Table 4. Radii derived with the current CORS method compared with previous determinations (see text).

\begin{tabular}{|c|c|c|c|c|c|c|c|c|}
\hline Cepheid & Binary & $\begin{array}{c}\text { Period } \\
\text { days } \\
\end{array}$ & $\begin{array}{c}R_{\text {without } \Delta B} \\
\frac{R}{R_{\odot}}\end{array}$ & $\begin{array}{c}R_{\Delta B} \\
\frac{R}{R_{\odot}} \\
\end{array}$ & $\begin{array}{c}R_{\mathrm{AFR}} \\
\frac{R}{R_{\odot}} \\
\end{array}$ & $\begin{array}{c}R_{\mathrm{LS}} / R_{\mathrm{GFG}} \\
\frac{R}{R_{\odot}}\end{array}$ & $\begin{array}{c}R_{\mathrm{RBMR}} . \\
\frac{R}{R_{\odot}} \\
\end{array}$ & $\begin{array}{c}R_{\mathrm{BM}} \\
\frac{R}{R_{\odot}} \\
\end{array}$ \\
\hline $\mathrm{FF} \mathrm{Aql}^{*}$ & $\mathrm{O}$ & 4.470840 & 49.8 & 54.1 & 52.0 & - & - & - \\
\hline FM Aql & - & 6.114334 & 56.5 & 59.3 & 59.3 & - & 60.6 & 51.61 \\
\hline FN Aql & - & 9.481614 & 83.5 & 86.5 & - & - & - & - \\
\hline$\eta \mathrm{Aql}$ & B & 7.176779 & 48.2 & 52.6 & 67.5 & - & 56.7 & 52.76 \\
\hline TT Aql & - & 13.75551 & 84.3 & - & 76.1 & - & 95.3 & 94.31 \\
\hline U Aql & $\mathrm{O}$ & 7.024100 & 45.4 & 47.0 & 48.9 & - & 61.3 & 52.45 \\
\hline V496 Aql & B & 6.807164 & 47.7 & 61.2 & 38.1 & - & 36.4 & 43.69 \\
\hline V600 Aql & - & 7.238748 & 55.4 & 80.1 & 56.3 & - & - & - \\
\hline RX Aur & B: & 11.623537 & 83.7 & 81.0 & - & - & - & 61.17 \\
\hline RT Aur & - & 3.728561 & 27.9 & 30.6 & 36.2 & - & 42.2 & 35.74 \\
\hline$\delta$ Cep & V & 5.366316 & 41.0 & 47.2 & 46.2 & - & 52.8 & 39.84 \\
\hline DT Cyg* & - & 2.499086 & 46.4 & 38.7 & 32.3 & - & 42.9 & - \\
\hline SU Cyg & $\mathrm{O}$ & 3.845733 & 35.4 & 40.8 & 48.9 & - & 61.3 & 52.45 \\
\hline X Cyg & - & 16.386130 & 104.1 & - & 69.3 & - & 96.2 & 114.29 \\
\hline VZ Cyg & $\mathrm{O}$ & 4.864504 & 45.3 & 53.1 & 35.8 & - & 47.3 & 35.57 \\
\hline BG Cru* & - & 3.342503 & 28.9 & 32.7 & - & - & - & - \\
\hline$\zeta \mathrm{Gem}$ & V & 10.150780 & 64.4 & 75.0 & 55.1 & - & 86.2 & 62.55 \\
\hline W Gem & - & 7.913960 & 96.9 & 86.4 & 48.5 & - & 60.7 & 48.69 \\
\hline BG Lac & $\mathrm{b}$ & 5.331938 & 47.0 & 48.9 & - & - & 35.8 & 43.49 \\
\hline X Lac & $\mathrm{b}$ & 5.444990 & 41.3 & 46.9 & 36.7 & - & 71.2 & 61.97 \\
\hline Z Lac & $\mathrm{O}$ & 10.88554 & 67.2 & 69.4 & 83.1 & - & 86.8 & 66.40 \\
\hline BF Oph & $\mathrm{b}$ & 4.067695 & 39.9 & 44.8 & - & $36.11^{a} / 35.8^{b}$ & 32.4 & 33.78 \\
\hline Y Oph & $\mathrm{b}$ & 17.126780 & 96.3 & 93.4 & - & $93.22^{a}$ & 112.2 & - \\
\hline AW Per & $\mathrm{O}$ & 6.463589 & 29.7 & 37.5 & 34.7 & - & 54.8 & 45.40 \\
\hline V440 Per* & B: & 7.572712 & 62.9 & 76.8 & - & - & - & - \\
\hline CM Sct & - & 3.916977 & 38.4 & 38.6 & 29.6 & - & - & - \\
\hline EV Sct & B: & 3.090998 & 30.9 & 42.0 & - & $47.62^{a} / 32.5^{b}$ & 36.6 & - \\
\hline SS Sct & - & 3.671280 & 39.6 & 44.2 & 30.4 & - & - & 27.71 \\
\hline RU Sct & - & 19.70062 & 102.9 & - & - & $120.45^{a}$ & - & - \\
\hline Y Sct & $\mathrm{b}$ & 10.341650 & 60.9 & - & - & - & 71.5 & 80.45 \\
\hline AP Sgr & B: & 5.0574269 & 60.4 & 62.3 & 36.6 & - & 50.0 & - \\
\hline S Sge & $\mathrm{O}$ & 8.382044 & 75.1 & 79.1 & 64.6 & - & - & - \\
\hline BB Sgr & - & 6.637115 & 47.6 & 61.3 & - & $37.39^{a} / 44.4^{b}$ & 53.5 & 40.42 \\
\hline U Sgr & - & 6.745363 & 76.6 & 82.5 & - & $52.30^{a} / 48.8^{b}$ & - & 57.61 \\
\hline V350 Sgr & $\mathrm{O}$ & 5.154557 & 32.1 & 32.7 & 40.2 & - & 46.0 & 49.55 \\
\hline X Sgr & - & 7.012630 & 52.5 & 59.8 & 68.5 & - & 58.6 & 47.76 \\
\hline Y Sgr & B & 5.773400 & 47.4 & 56.2 & - & - & 61.4 & 47.95 \\
\hline YZ Sgr & - & 9.553606 & 93.6 & 95.8 & - & - & 136.0 & - \\
\hline W Sgr & $\mathrm{O}$ & 7.595080 & 58.6 & 62.6 & 62.5 & - & 58.6 & 60.77 \\
\hline WZ Sgr & B & 21.849708 & 120.5 & - & - & $121.43^{a} / 122.2^{b}$ & - & 151.20 \\
\hline SV Vul & $\mathrm{B}$ & 44.999660 & 213.0 & - & 135.6 & $241.62^{a} / 250.7^{b}$ & - & 189.5 \\
\hline $\mathrm{T}$ Vul & $\mathrm{b}$ & 4.435532 & 31.8 & 37.1 & 42.3 & - & 48.8 & 36.54 \\
\hline U Vul & $\mathrm{O}$ & 7.990736 & 75.0 & 84.4 & 57.9 & - & 61.1 & 54.34 \\
\hline
\end{tabular}

An asterisk at top-right of Cepheid name means that it pulsates as a First Overtone.

In the second column: B - spectroscopic binary, B: - spectroscopic binary but confirmation needed; b - Photometric companion, physical relation should be investigated; O - spectroscopic binary with known orbit; V visual binary (see Szabados 2003 for details).

Radius sources: $R_{\mathrm{AFR}}$ Arellano Ferro \& Rosenzweing (2000); $R_{\mathrm{LS}}$ Laney \& Stobie (1995) / $R_{\mathrm{GFG}}$ Gieren et al. (1998): in the table ${ }^{a}$ refer to $R_{\mathrm{LS}}$ and ${ }^{b}$ to $R_{\mathrm{GFG}} ; R_{\mathrm{RBMR}}$ Ripepi et al. (1997); $R_{\mathrm{BM}}$ Barnes $\&$ Moffet (1987). 
Table 5. Comparison with radii measured with interferometric techniques (see text for details).

\begin{tabular}{cccc}
\hline \hline Star & $R_{I}$ & $R_{\text {without } \triangle B}$ & $R_{\text {with } \Delta B}$ \\
\hline$\eta$ Aql & $58.8 \pm 7.6$ & 48.2 & 52.6 \\
$\delta$ Cep & $47_{-6}^{+8}$ & 41.0 & 47.2 \\
$\zeta$ Gem & $63.4 \pm 7.2$ & 64.4 & 75.0 \\
\hline
\end{tabular}

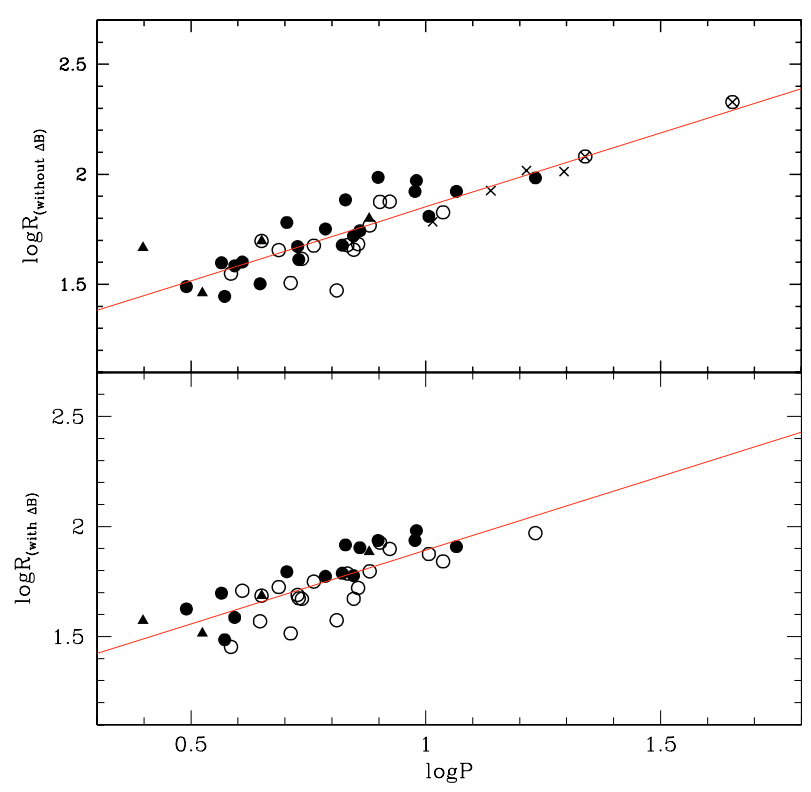

Fig. 13. Top: Period-Radius relation obtained in the case without $\Delta B$; Bottom: as before, but with $\Delta B$. Open circle: Binary stars; Triangles: first overtone pulsators; Crosses: stars with loop in the $\left[m_{1}\right],\left[c_{1}\right]$ plane not completely within the theoretical grids (see Sect. 3).

on the radii found, we conclude that our results are globally consistent with the interferometric ones.

\section{The Period-Radius relation}

After having tested the method and the consistency of our results with previous determinations in the literature, we are in the position to derive a PR relation based on the new derived radii.

The PR relations for all the stars in our sample for which the program reached the convergence are shown in Fig. 13, where the the top and bottom panels illustrate the case without and with $\Delta B$ respectively. A least square fit to the data (solid lines in Fig. 13) leads to the following Period-Radius relations in the case without the $\Delta B$ term (Eq. (12)) and with the $\Delta B$ term (Eq. (13)):

$$
\begin{array}{ll}
\log R=(1.18 \pm 0.05)+(0.67 \pm 0.06) \log P & \mathrm{rms}=0.09 \\
\log R=(1.22 \pm 0.06)+(0.67 \pm 0.08) \log P & \mathrm{rms}=0.08
\end{array}
$$

An inspection of the figure suggests that the presence of first overtone pulsators (filled triangles) could affect the derived PR relations because, at fixed period, they are brighter and, in turn, have larger radii than fundamental pulsators.

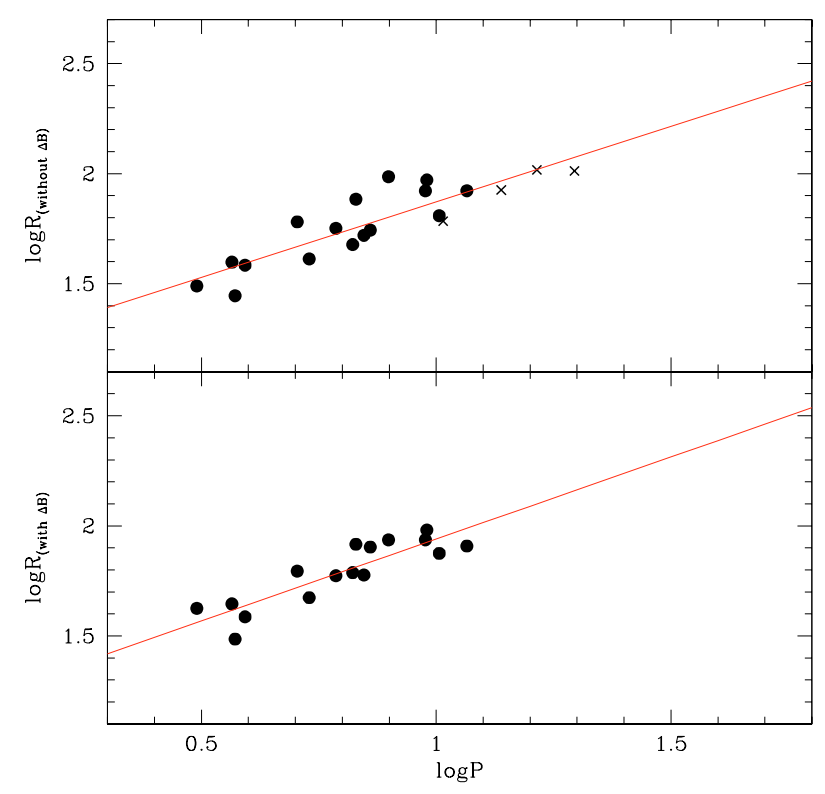

Fig. 14. The same of Fig. 13 but excluding first overtone and binary stars.

Similarly, also Cepheids belonging to binary systems could affect the radius determination. Therefore, we decided to exclude from our relations the first overtone pulsators and the stars flagged as "B" and "O"3 in Table 4, whereas we left in our sample the Cepheids flagged as "B:", "b" and "V" (i.e. uncertain spectroscopic binaries and separated visual binaries respectively, see Szabados 2003). As a result of this selection procedure, we are left with 20 and 16 Cepheids in the cases without and with $\Delta B$ respectively. We therefore calculated new Period-Radius relations with the following results (see Fig. 14):

$$
\begin{array}{ll}
\log R=(1.18 \pm 0.08)+(0.69 \pm 0.09) \log P & \mathrm{rms}=0.08 \\
\log R=(1.19 \pm 0.09)+(0.74 \pm 0.11) \log P & \mathrm{rms}=0.07
\end{array}
$$

A comparison between Eqs. (12), (13) and Eqs. (14), (15) shows that the net result of our selection criterion mainly consists in increasing the slope of the PR relation, going in the direction to improve the agreement with the literature, and, in particular with the NIR results by Laney \& Stobie (1995) and Gieren et al. (1998) (see Table 6 for a comparison between present results and other relations in the literature). Yet, we have to note that the errors of the derived coefficients are rather large. This is caused by: 1) the small number of Cepheids left after the selection process (in particular the number of binary stars in our sample is very large); 2) the lack of long period Cepheids in the case with $\Delta B$, as a result of problems with synthetic model atmosphere grids (see Sect. 3.1). We remark, however, that including or not the $\Delta B$ term in the CORS determination makes some difference, in the sense that, apart from a few Cepheids in the low period range, the scatter in the PR relation is slightly reduced (see Fig. 14) when the $\Delta B$ term is included.

\footnotetext{
3 We tried to determine the radius for the few Cepheids with known orbits, by disentangling orbital motions and pulsation in the radial velocity curve, but the error produced in this procedure remained still too high to secure good results.
} 
Table 6. Comparison between the PR $(\log R=a \log P+b)$ coefficients obtained in this paper and the ones based on selected works in literature. In particular, from left to right, we report the slope, the zero point, the source and the method adopted for deriving the PR.

\begin{tabular}{llll}
\hline \hline$a$ & $b$ & Source & Method \\
\hline $0.74 \pm 0.03$ & $1.12 \pm 0.03$ & RAAF & Surf. brightness \\
$0.751 \pm 0.026$ & $1.070 \pm 0.008$ & LS & Surf. brightness \\
$0.750 \pm 0.024$ & $1.075 \pm 0.007$ & GFG & Surf. brightness \\
$0.606 \pm 0.037$ & $1.263 \pm 0.033$ & RBMR & CORS \\
$0.655 \pm 0.006$ & $1.188 \pm 0.008$ & BCM & Theory \\
$0.69 \pm 0.09$ & $1.18 \pm 0.08$ & This work & new CORS (without $\Delta B$ ) \\
$0.74 \pm 0.11$ & $1.19 \pm 0.09$ & This Work & new CORS (with $\Delta B)$ \\
\hline
\end{tabular}

PR source: Rojo Arellano \& Arellano Ferro (1994, RAAF); Laney \& Stobie (1995, LS); Gieren et al. (1998, GFG); Ripepi et al. (1997, RBMR); Arellano Ferro \& Rosenzweig (2000, AFR); Bono et al. (1998, BCM).

Table 7. Top: observed properties of Y Oph; Bottom: physical parameters of the best fit model.

\begin{tabular}{cccccc}
\hline \hline$P$ & $\left\langle m_{V}\right\rangle$ & $B-V$ & $Z$ & $E(B-V)$ & $A$ \\
\hline 17.1268 & 6.169 & 1.377 & 0.05 & 0.655 & 0.483 \\
\hline$M$ & $Y$ & $Z$ & $T_{\text {eff }}$ & $\frac{R}{R_{\odot}}$ & $\log \frac{L}{L_{\odot}}$ \\
\hline $7 M_{\odot}$ & 0.28 & 0.02 & $4720 \mathrm{~K}$ & 97.77 & 3.64 \\
\hline
\end{tabular}

This result seems to suggest that the modification to the CORS method presented in this paper is well suited also when applied to real stars (not merely to synthetic light curves).

Finally, we notice that our results concerning the radii and the PR relations could be, in principle, combined with an effective temperature calibration to derive the intrinsic stellar luminosity, through the Stefan-Boltzmann law, and in turn distance to studied Cepheids. This possibility will be investigated in a forthcoming paper. In the following section we will apply an alternative method to derive the luminosity and distance by means of the comparison of empirical light and radial velocity curves with the predictions of the nonlinear convective pulsation models discussed above.

\subsection{Theoretical fit of the light and radial velocity curves of the Cepheid Y Oph}

Before going to the conclusions of this paper, we note that it has recently been suggested that nonlinear pulsation models provide a direct tool to evaluate the intrinsic stellar properties of pulsating stars through the comparison of observed and predicted variations of relevant parameters along a pulsation cycle (see Wood et al. 1997; Bono et al. 2000, 2002). This kind of analysis also provides an additional test for our radius determination technique by means of the comparison with the radius of the model which is able to simultaneously reproduce the period, the amplitude and the morphology of light and radial velocity curves. We plan to apply this method to a sample of Galactic Cepheids with accurate photometric data and available radial velocity information. In this paper we present a first application to the Cepheid Y Oph. The observed properties of this star are summarized in Table 7.

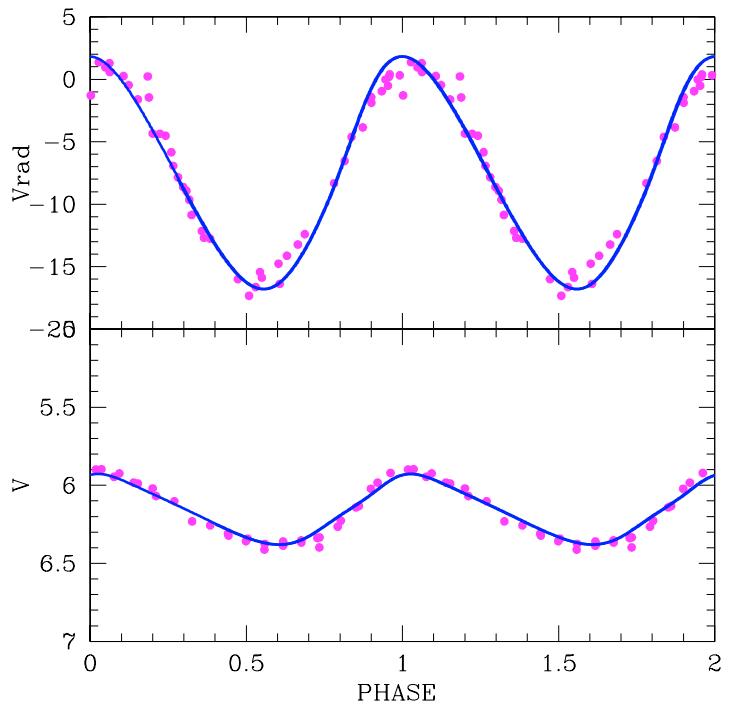

Fig. 15. Top panel: empirical light curve (dots) for the star Y Oph, compared with the best fit model (solid lines, see text for details). Bottom panel: the same but for the radial velocity curve.

Starting from the observed radial velocity and light curve, we try to reproduce their morphology and amplitude, by computing pulsation models along isoperiodic (with period equal to the observed one) sequences with varying pulsation mass and effective temperature and assuming, for each mass, a canonical mass-luminosity relation. The best fit model resulting from these computations is shown in Fig. 15 (solid line). The corresponding stellar parameters are reported in Table 7 and show that the agreement with the radius determined in this paper is good.

The other important information provided by the fit is the stellar absolute magnitude $M_{V}=-3.996 \mathrm{mag}$, which allows us to estimate a distance of about $423 \mathrm{pc}$, consistent with the independent evaluation by Gieren et al. (1993), thus supporting the predictive capability of pulsation models.

\section{Final remarks}

We have presented a modified version of the CORS method based on a new calibration of the Surface Brightness function in 
Table A.1. Coefficients for the polynomial fits described in the Appendix.

\begin{tabular}{rrrrrrrrr}
\hline \hline$a_{0}$ & $a_{1}$ & $a_{2}$ & $a_{3}$ & $a_{4}$ & $a_{5}$ & $a_{6}$ & $a_{7}$ & $a_{8}$ \\
3.8911 & -0.4273 & 0.1806 & 0.2168 & -0.7049 & 0.5227 & -0.0609 & 0.3989 & \\
\hline$b_{0}$ & $b_{1}$ & $b_{2}$ & $b_{3}$ & $b_{4}$ & $b_{5}$ & $b_{6}$ & $b_{7}$ & $b_{8}$ \\
2.4 & 11.7 & -7.1 & 10.4 & -47.1 & -3.5 & 39.9 & & \\
\hline$c_{0}$ & $c_{1}$ & $c_{2}$ & $c_{3}$ & $c_{4}$ & $c_{5}$ & $c_{6}$ & $c_{7}$ & $c_{8}$ \\
-276.874 & 144.304 & -18.803 & 76.782 & -40.744 & 5.403 & -15.724 & 8.378 & -1.116 \\
\hline
\end{tabular}

the Strömgren photometric system. In particular we have been able to derive a calibration of $S_{V}$ as a function of the Strömgren reddening free indexes $\left[m_{1}\right]$ and $\left[c_{1}\right]$ by adopting grids of theoretical colors. This procedure revealed the unexpected occurrence that the quoted theoretical grids are not able to fully include the location of actual long period ( $P \geq 12-13$ days) Cepheids loops in the $\left[m_{1}\right],\left[c_{1}\right]$ plane. This problem could only be overcome by adopting next generation, hopefully improved, model atmosphere grids. Nevertheless, the modified CORS method presented here has been tested by means of synthetic light and radial velocity curves derived from nonlinear pulsation models and simulations have been performed to take into account the quality of real observed curves as well as possible time shifts between photometric and radial velocity data. The results of such tests can be summarized as follows:

a) The present method appears capable to derive the radius of a Cepheid with an average precision of around 3.5\% and 5\% in the case of good and fair observational data respectively (see Sect. 4.3).

b) The error associated with a phase shift between photometric and radial velocity data could be as large as $\sim 17 \%$ in the extreme case of $\Delta \phi \sim 0.05$. In a more common case of $\Delta \phi \sim$ 0.01 the relative error is still rather large, namely $\sim 5 \%$. If we add to the error budget the uncertainty on the value of the projection factor $k(\sim \pm 2 \%$ for $\Delta k \pm 0.03)$, neglecting other possible contributions (see Bono et al. 1994 and references therein for a detailed discussion), we can conclude that estimating the radius of a Cepheid by means of the Baade-Wesselink method (at least in the present formulation) with an accuracy better than $10 \%$ is extremely difficult to be achieved.

The method has been then applied to a sample of Galactic Cepheids with Strömgren photometry and radial velocity data to derive the radii and a new PR relation. As a result we obtained the following PR relation: $\log R=(1.19 \pm 0.09)+(0.74 \pm$ $0.11) \log P(\mathrm{rms}=0.07)$. This relation, even if not very accurate (mainly due to the unexpected presence of a large fraction of binary stars in our Cepheid sample), nevertheless is in satisfactory agreement with previous findings in the literature.

Acknowledgements. We wish to thank our anonymous referee for several pertinent suggestions that improved the content and the readability of the paper. This work made use of the "McMaster Cepheid Photometry and Radial Velocity Data Archive" maintained by Doug Welch, and of SIMBAD database, maintained at the CDS-Strasbourg. This work was partially supported by MIUR/Cofin 2002, under the project "Stellar Populations in Local Group Galaxies" (Monica Tosi coordinator).

\section{Appendix A:}

The 4th degree polynomial fit to effective temperature, effective gravity, and bolometric corrections mentioned in Sect. 3.1.1 are the following:

$$
\begin{aligned}
\log T_{\mathrm{eff}}= & a_{0}+a_{1} \cdot\left[m_{1}\right]+a_{2} \cdot\left[m_{1}\right]^{2}+a_{3} \cdot\left[c_{1}\right] \\
& +a_{4} \cdot\left[m_{1}\right] \cdot\left[c_{1}\right]+a_{5} \cdot\left[m_{1}\right]^{2} \cdot\left[c_{1}\right]+a_{6} \cdot\left[c_{1}\right]^{2} \\
& +a_{7} \cdot\left[m_{1}\right]^{2} \cdot\left[c_{1}\right]^{2} \\
\log g_{\mathrm{eff}}= & b_{0}+b_{1} \cdot\left[m_{1}\right]+b_{2} \cdot\left[m_{1}\right]^{2}+b_{3} \cdot\left[c_{1}\right] \\
& +b_{4} \cdot\left[m_{1}\right] \cdot\left[c_{1}\right]+b_{5} \cdot\left[c_{1}\right]^{2} \\
& +b_{6} \cdot\left[m_{1}\right]^{2} \cdot\left[c_{1}\right]^{2} \\
B C= & c_{0} \cdot \log T_{\mathrm{eff}}+c_{1} \cdot \log T_{\mathrm{eff}}^{2} \\
+ & c_{2} \cdot \log g_{\mathrm{eff}}+c_{3} \cdot \log T_{\mathrm{eff}} \cdot \log g_{\mathrm{eff}} \\
& +c_{4} \cdot \log T_{\mathrm{eff}}^{2} \cdot \log g_{\mathrm{eff}}+c_{5} \cdot \log g_{\mathrm{eff}}^{2} \\
+ & c_{6} \cdot \log T_{\mathrm{eff}} \cdot \log g_{\mathrm{eff}}^{2}+c_{7} \cdot \log T_{\mathrm{eff}}^{2} \cdot \log g_{\mathrm{eff}}^{2}
\end{aligned}
$$

the coefficients $a_{i}, b_{i}, c_{i}$ of the previous relations are listened in Table A.1 and the other symbols have their usual meaning. Note the rms of the previous relations are $0.0018 \mathrm{dex}, 0.1 \mathrm{dex}$ and 0.003 mag respectively.

\section{References}

Antonello, E., Poretti, E., \& Reduzzi, L. 1990, A\&A, 236, 138

Arellano Ferro, A., Rojo Arellano, E., Gonzàlez-Bedolla, S., \& Rosenzweig, P. 1998, ApJ, 117, 167 (AFGR)

Arellano Ferro, A., \& Rosenzweing, P. 2000, MNRAS, 315, 296 (AFR)

Baade, W. 1926, Astron. Nachr., 228, 359

Barnes, T. G., Evans, D. S., \& Parson, S. B. 1976, MNRAS, 174, 489

Barnes, T. G., Moffet, T. J., \& Slovak, M. H. 1987, ApJS, 65, 307 (BMS)

Barnes, T. G., Moffet, T. J., \& Slovak, M. H. 1988, ApJS, 66, 43 (BMS)

Bersier, D., Burki, G., Mayor, M., \& Duquennoy, A. 1994, A\&AS, 108, 25 (BBMD)

Bono, G., Caputo, F., \& Marconi, M. 1998, ApJ, 497, L43 (BCM)

Bono, G., Caputo, F., \& Stellingwerf, R. F. 1994, ApJ, 432, L51

Bono, G., Castellani, C., \& Marconi, M. 2000, ApJ, 532, L129

Bono, G., Marconi, M., \& Stellingwerf, R. F. 1999a, ApJS, 122, 167 (BMS99)

Bono, G., Gieren, W. P., Marconi, M., \& Fouqué, P., Caputo, F. 2001, ApJ, 563, 319

Bono, G., Castellani, V., \& Marconi, M. 2002, ApJ, 565, L83 
Caccin, R., Onnembo, A., Russo, G., \& Sollazzo, C. 1981, A\&A, 97, 104

Castellani, V., Chieffi, A., \& Straniero, O. 1992, ApJS, 78, 517

Castelli, F., Gratton, R. G., \& Kurucz, R. L. 1997a, A\&A, 318, 841 (C97a)

Castelli, F., Gratton, R. G., \& Kurucz, R. L. 1997b, A\&A, 324, 432 (C97b)

Coulson, I. M., Caldwell, A. R., \& Gieren, W. P. 1985, ApJS, 57, 595 (CCG)

Crawford, D. L., \& Mandwewala, N. 1976, PASP, 88, 917

Eggen, O. J. 1985, AJ, 90, 1297 (Eg)

Eggen, O. J. 1996, AJ, 111, 1313 (Eg)

Evans, L. T. 1980, SAAO Circulars 1, 257 (E)

Feltz, K. A., \& McNamara, D. H. 1980, ASP, 92, 609 (FM)

Freedman, W. L., Madore, B. F., \& Kennicutt, R. C. 1997, in The Extragalactic Distance Scale, ed. M. Livio, M. Donahue, \& N. Panagia (Cambridge: Cambridge Univ. Press), 171

Freedman, W. L., Madore, B. F., Gibson, B. K., et al. 2001, ApJ, 553, $47 \mathrm{~F}$

Gieren, W. P. 1981, ApJS, 46, 287 (G)

Gieren, P. W., Barnes III, T. G., \& Moffett, T. J. 1993, ApJ, 418, 135

Gieren, P. W., Fouqué, P., \& Gómez, M. 1997, ApJ, 488, 74

Gieren, P. W., Fouqué, P., \& Gómez, M. 1998, ApJ, 496, 17 (GFG)

Gorynya, N. A., Samus, N. N., SachKov, M. E., et al. 1998, Astron. Lett., 24, 6, 815 (GSSRGA)

Gray, R. O. 1991, A\&A, 252, 237

Imbert, M. 1999, A\&AS, 140, 791 (I)

Kiss, L. L. 1998, MNRAS, 297, 825 (K)
Lane, B. F., Creech-Eakman, M. J., \& Nordgren, T. E. 2002, ApJ, 124, 541

Laney, C. D., \& Stobie, R. S. 1995, MNRAS, 274, 337 (LS)

Marconi, M., Caputo, F., Di Criscienzo, M., \& Castellani, M. 2003, ApJ, 596, 299

Metzger, M. R., Caldwell, J. A. R., McCarthy, J. K., \& Schechter, P. L. 1993, ApJS, 76, 803 (MCMS)

Moffett, T. J., \& Barnes, T. J., III 1987, ApJ, 323, 280

Moskalik, P. 2000, in The Impact of Large-Scale Surveys on Pulsating Star Research, ed. L. Szabados, \& D. W. Kurtz, IAU Coll., 176, ASP, 233

Onnembo, A., Buonaura, B., Caccin, B., Russo, G., \& Sollazzo, C. 1985, A\&A, 152, 349

Nordgren, T. E., Armstrong, J. T., German, M. E., et al. 2000, ApJ, 543, 972

Pardo, I., \& Poretti, E. 1997, A\&A, 324, 121

Pel, J. W. 1978, A\&A, 62, 75

Ripepi, V., Barone, F., Milano, F., \& Russo, G. 1997, A\&A, 318, 797 (RBMR)

Ripepi, V., Russo, G., Bono, G., \& Marconi, M. 2000, A\&A, 354, 77

Rojo Arellano, E., \& Arellano Ferro, A. 1994, RMxAA, 29, 148 (RAAF)

Sollazzo, C., Russo, G., Onnembo, A., \& Caccin, B. 1981, A\&A, 152, 3490

Szabados, L. 2003, INf. Bull. Var. Stars, 5394

Wilson, T. D., Carter, M. W., Barnes, T. G., Van Citters, G. W., \& Moffett, T. J. 1989, ApJS, 69, 951 (WCB)

Wood, P. R., Arnold, A., \& Sebo, K. M. 1997, ApJ, 485, L25 